\title{
Rapid Screening of Guar Gum Using Portable Raman
}

\section{Spectral Identification Methods}

\author{
Hirsch K. Srivastava, Steven Wolfgang and Jason D. Rodriguez * \\ Division of Pharmaceutical Analysis, Center for Drug Evaluation and Research, US Food and Drug \\ Administration, 645 S. Newstead Ave St. Louis, MO 63110 \\ * EMAIL: Jason.Rodriguez@fda.hhs.gov
}

CORRESPONDING AUTHOR INFORMATION: Tel (314) 539-3855 Fax (314) 539-2113 


\section{ABSTRACT}

Guar gum is a well-known inactive ingredient (excipient) used in a variety of oral pharmaceutical dosage forms as a thickener and stabilizer of suspensions and as a binder of powders. It is also widely used as a food ingredient in which case alternatives with similar properties, including chemically similar gums, are readily available. Recent supply shortages and price fluctuations have caused guar gum to come under increasing scrutiny for possible adulteration by substitution of cheaper alternatives. One way that the U.S. FDA is attempting to screen pharmaceutical ingredients at risk for adulteration or substitution is through field-deployable spectroscopic screening. Here we report a comprehensive approach to evaluate two field-deployable Raman methods - spectral correlation and principal component analysis - to differentiate guar gum from other gums. We report a comparison of the sensitivity of the spectroscopic screening methods with current compendial identification tests. The ability of the spectroscopic methods to perform unambiguous identification of guar gum compared to other gums makes them an enhanced surveillance alternative to the current compendial identification tests, which are largely subjective in nature. Our findings indicate that Raman spectral identification methods perform better than compendial identification methods and are able to distinguish guar gum from other gums with $100 \%$ accuracy for samples tested by spectral correlation and principal component analysis.

\section{Keywords:}

Rapid screening, economically-motivated adulteration, raw ingredients, spectroscopy, identification tests 


\section{INTRODUCTION}

Guar gum is a water-soluble polysaccharide composed of the sugars galactose and mannose and is based on a $\beta$ 1,4-linked mannose backbone to which galactose residues are 1,6-linked at every second mannose[1]. Guar gum produces a highly viscous, thixotropic solution and is obtained from the grinding of endospores of the seed of the leguminous tree Cyamopsis tetragonlubus located mostly in the Indian subcontinent. In tablet manufacturing guar gum is used as a binder and disintegrating agent and in micro-encapsulation of drugs[2].

Since 2009, FDA has applied a working definition of economically-motivated adulteration (EMA) as "the fraudulent, intentional substitution or addition of a substance in a product for the purpose of increasing the apparent value of the product or reducing the cost of its production, i.e., for economic gain[3] ." Documented incidents of EMA involve a common vulnerability, i.e., the substitutes possess similar physical and/or chemical properties and are thus difficult to detect by non-specific compendial and conventional tests used by industry[4-9]. Recent high profile cases of EMA include the 2008 heparin crisis due to contamination of raw heparin stock imported from China[4] and the 2007-2008 melamine contaminations of pet food, milk and infant formula. Guar gum's use as a chemical additive for a rapidly expanding petroleum gas fracking industry in the US may have contributed to a recent guar gum shortage and an observed 500\% price increase during a period of 15 months beginning in $2012[10$, 11]. These events have further raised concerns about vulnerability towards possible substitution of guar gum by cheaper gum alternatives.

In 2012, the U.S. FDA alerted the United States Pharmacopeia (USP) that guar gum was one of 12 excipients considered at elevated risk of adulteration due to an ambiguous identification (ID) test. The ID test lacks specificity, and analysis of a sample from a container labeled guar gum could result in a false positive identification for a mixture containing guar gum. Shortly after being notified by FDA, USP initiated a project to revise the ID test for guar gum NF to include a qualitative determination of presence of the main sugar components, galactose and mannose, by thin layer chromatography[12]. 
This improvement in the ID test still leaves some uncertainty about the total composition and whether other galactomannans are present[13].

Manufacturers of finished pharmaceuticals are generally accustomed to relying on a supplier certificate of analysis and performing an ID test in order to release incoming shipments of excipients such as guar gum. However, most ID tests lack the specificity and sensitivity to be effective in screening for possible adulteration. In addition, many of the analytical ID test methods are time consuming, require complex sample preparation and are designed for use in the laboratory setting[14]. There are currently two compendial tests to confirm the identity and purity of guar gum: a colorimetric ID test (Test A) and a thin-layer chromatography test (Test B). The current USP/NF compendial method for ID of Guar Gum (ID Test A) has an acceptance criteria that includes an "appreciable" change in viscosity and a change to produce an "opalescent" solution[15]. Using such a subjective and qualitative test, however, has well-known limitations in differentiating guar gum from locust bean gum, gellan gum, and other galactomannans due to the similarity in their compositions[9]. More specific analytical methods based on differences in galactomannan composition remain viable for identification of guar gum and differentiation from similar galactomannans. These include chromatography or electrophoresis and hydrolysis[16] with subsequent determination of the monosaccharide composition; the latter is the new USP ID Test B[12]. These analytical methods are destructive to the sample, timeconsuming, and are relegated to the laboratory setting. Thus, these methods are not amenable to tests designed to rapidly screen ingredients and identify those which are either falsely labeled or which possibly contain an adulterant.

In this paper we propose an improved ID test that is based on spectral library based methods. Spectroscopic methods are non-destructive, require minimal sample preparation, and can be carried out on portable instruments at the pharmaceutical product manufacturing facility by non-experts. Previous studies have indicated that they are capable of screening for and detecting adulterant [17] levels which are in the $5-25 \%$ range[18]. Raman spectroscopic techniques are often used to acquire the unique vibrational molecular signature of substances. Until recently, Raman was regarded as primarily a 
laboratory method due to the large footprint required for traditional Raman spectrometers. Today, miniaturization of the optical and electronic components used to craft these instruments has led Raman to be commonly available in portable/handheld platforms from a variety of commercial vendors[19]. One of the greatest benefits of using these instruments is their ability to perform rapid interrogation of the sample under study, often in its original packaging, without any additional sample preparation[18]. Raman instruments can be programmed with methods ranging from correlation-based spectral library tests[20] to more rigorous multivariate-based tests such as principle component analysis (PCA)[21, 22]. Both approaches were used in this study to evaluate the ability of spectroscopic methods to provide unambiguous ID of guar gum versus monograph methods.

\section{MATERIAL AND METHODS}

2.1 Sample Preparation. The gum samples used to in this work were taken from commerciallyavailable samples from various manufacturers and lots without further purification. A total of 18 different samples were used for method development - ten guar gum samples and eight other gums. The samples are listed in Table 1.

2.2 Compendial Identification Tests. The different gum samples were tested according to the ID Test A compendial identification test for guar gum[15]. Briefly, approximately 2 grams of sample were placed into a $400 \mathrm{~mL}$ beaker and moistened with $4 \mathrm{~mL}$ of isopropyl alcohol. Next, $200 \mathrm{~mL}$ of cold water were added to the sample and stirred vigorously until the sample was uniformly dispersed. An authentic guar gum sample prepared in this manner should result in in an opalescent (off white) viscous solution. A $100 \mathrm{~mL}$ portion of the solution is then heated in a bath of boiling water for about 10 minutes. Authentic guar gum samples should exhibit no appreciable increase in viscosity.

2.3 Spectral Collection. All Raman spectra were collected through clear polyethylene bags. Spectra were acquired on an EZ-Raman I Raman Spectrometer (Enwave Optronics Inc.) using the output of a $785 \mathrm{~nm}$ laser $(340-\mathrm{mW})$. The TE-cooled CCD detector was operated at $-50^{\circ} \mathrm{C}$ with variable acquisition times. The resolution of the spectrometer is $\sim 6 \mathrm{~cm}^{-1}$ and uses a sampling spot size $\sim 200 \mu \mathrm{m}$. Each sample bag was measured 15 times using random sampling at different locations on the bag. Raman 
spectra for all guar gum and gum samples were collected in the spectral range of $250-2400 \mathrm{~cm}^{-1}$. The spectral range used for method development, described in the next section, was $300-2000 \mathrm{~cm}^{-1}$.

2.4 Algorithm/Model Construction. Two types of spectroscopic models were used in this work: 1) spectral library spectral correlation and 2) PCA. The library spectrum for each gum was obtained by taking the average spectrum of 15 different measurements from random location on the bag. The spectral correlation values (also known as hit quality index [HQI]) reported in this study were calculated using the spectral correlation algorithm and is shown in Equation 1,

$$
S C=\frac{(\text { Library } \cdot \text { Test })^{2}}{(\text { Library } \cdot \text { Library })(\text { Test } \cdot \text { Test })}
$$

where Library indicates the library spectrum and Test indicates an individual spectrum of the material under study. The Library spectrum used for all SC studies was the composite (average) spectrum for all ten guar gum samples included in the study. The SC defined in Equation 1 is the square of the spectral covariance between the library and test spectra normalized by the squared norms of the two spectral vectors (i.e., the square of the spectral correlation coefficient). Values for SC range from 1.000, which indicates perfect correlation and 0 , which indicates poor correlation. The threshold used for a Pass was set to 0.95. First derivative preprocessing[23] (Gram Polynomial $2^{\text {nd }}$ order 15 point window) was applied to all spectra prior to calculating the SC value. PCA data analysis was performed using PLS Toolbox (Version 8.0.2) carried out in MATLAB (Version 8.6, R2015b). The same 15 replicate spectra of each gum sample used for the SC method were also used to develop the classes for each gum for the PCA method. The library class for guar was the composite of all 10 guar gum samples (150 spectra). The preprocessing used to develop the PCA model was Savitzky-Golay first derivative $\left(2^{\text {nd }}\right.$ order, 15 point window) followed by vector length normalization. Cross validation (venetian blinds, 10 data splits) was used to help determine the appropriate amount of principal components for each model. The numbers of principal components chosen for the model were based on careful comparison between the principal component distributions and examination of the root mean squared error of calibration (RMSEC) trends and root mean squared error of cross validation (RMSECV) values. 


\section{RESULTS AND DISCUSSION}

The visual results of USP-NF compendial ID Test A for guar gum applied to all the gum samples listed in Table 2 are shown in Figure 1. Although opalescence and viscosity are impossible to show, the figure shows differences between the gums and the array of different appearances that may arise from such tests. Based solely on the visual results, it is apparent that the compendial ID Test A lacks specificity, with several gums appearing to be similar to the library sample. It should be noted that the two guar gum control samples are also not exactly the same in appearance. In Figure 1, the only sample that is clearly different is ghatti gum. Experimental results showed a similar appearance among solutions of guar gum, gellan gum, xanthan gum, tamarind gum and locust bean gum prepared as prescribed in USP ID Test A.

When considering the compendial criteria for viscosity, the specificity and sensitivity of the compendial ID test is dramatically improved. Based on this requirement, only control guar gum samples and gellan gum produce viscous solutions. The viscosity requirement is further confirmed upon boiling the samples, where the viscosity remained unchanged for these three samples. Therefore, three samples passed the USP-NF compendial identification method for guar gum and only two of those samples were actually guar gum. Thus, the compendial identification tests generally did an adequate job of differentiating guar from many other similar gums but were not able to adequately distinguish between gellan and guar gums, showing that gellan gum (and perhaps mixtures containing guar gum) may erroneously pass the existing guar gum identification tests.

The same samples for the various gums were also studied using portable Raman to gauge the effectiveness of spectral library-based identification methods. These spectral based identification methods eliminate the need for the analyst to make subjective decisions based on color and viscosity. The Raman spectra and first derivative spectra for guar gum and several other gums are shown in Figure 2. The average spectrum of all ten guar gum library samples was used to generate the library spectrum of guar gum for comparison with the other gums. This was done in order to create a representative reference spectrum of guar gum. The average SC for the set of ten guar gum library samples used to 
calculate the average reference spectrum of guar gum was $0.972 \pm 0.022$, indicating that the $\mathrm{SC}$ value is largely insensitive at detecting minor spectral differences between guar gum samples from different manufacturers and lots. Each of the other gum spectra shown in Figure 2 is the average of 15 replicate runs. As seen in Figure 2A, the unprocessed Raman spectra contain mostly broad features which make visual differentiation difficult. Two samples — rosin gum and ghatti gum — which were analyzed by the compendial ID test were not analyzed by Raman due to high levels of florescence in the spectra that saturated the Raman detector. Varying levels of fluorescence were present for all samples tested and is probably due primarily to colored impurities. The first derivative spectra for each of the gums are shown in Figure 2B. First derivative preprocessing assists in reducing the baseline due to fluorescence that is present in the spectra and has been shown to enhance spectral correlation tests[23]. The spectral correlation results shown above each spectrum in Figure 2B (and also listed in Table 2) indicate that all of the non-guar gum samples fail to meet the 0.95 Pass/Fail threshold.

To further investigate the utility of spectral based ID methods for differentiating guar gum from others gums, the Raman spectral results were additionally scrutinized by principal component analysis (PCA) methods. The PCA plot for the ten guar gum library samples is shown in Figure 3. There are two groupings of guar gum samples apparent in the plot. Generally, the plot indicates that different lots from the same manufacturer cluster together. The one exception are the three lots from Spectrum which has one lot that has a slightly different PCA distribution from the other two lots. The two samples from MP Biomedicals cluster together and are highly overlapped. This is not surprising since both are labeled under the same batch/lot number even though they were obtained in different packaging containers. The entire set of guar gum library spectra (10 library samples, 150 spectra) was combined to form a guar gum library class. This class was then compared to each of the six gums analyzed by Raman. The PCA plot comparing guar and other gums is shown in Figure 4. Each group of 15 spectra for each gum was designated as a separate class to create a PCA model. Each class is shown in Figure 4 surrounded by a 95\% confidence ellipsoid. As shown in Figure 4, no overlap is observed between the combined guar gum class and the classes from other gums. All other gums are clearly distinct from the 
guar gum library class. These results are significant because they show that a representative class with many library samples can be created for an excipient — in this case guar gum — without losing sensitivity for distinguishing between related substances (the other gums). One of the challenges in creating a large class with lot-to-lot and manufacturer/supplier variability built-in is that differences in authentic samples may lead to a loss of distinguishing ability of the PCA method for highly-similar or adulterated samples. This is because the variability built into the library class causes a broadening of the distribution of scores encompassed by the confidence ellipsoid. For the guar gum system, it is apparent that the variability in authentic guar gum samples is small compared to the differences observed between guar gum and other gums. While ten samples were used to generate the PCA class in this study, the number of samples in the library class may vary according to the system under consideration.

\section{CONCLUSIONS}

Compendial ID Test A was able to distinguish guar gum from seven other gums but failed to discern between guar gum and gellan gum. Such tests are "non-specific" in that they might not be capable of ruling out mixtures of guar gum with substances having similar physical properties. The subjective nature of tests based on appearance leaves ample room for interpretation depending on the user and thus raise the possibility of obtaining a false positive for a guar gum sample containing a substitute gum. Spectroscopic methods are a better choice for identification of guar gum for various reasons including reduced sample preparation and being non-destructive towards the sample. Using library-based SC methods, Raman is able to differentiate between guar gum and other galactomannan gums. The PCA results were essentially the same as the SC results and essentially reveal that spectroscopic methods are more effective than the current compendia ID Test A to detect whether a guar gum sample has undergone substitution with another gum. It is likely that the library-based SC methods would be easier to implement than the PCA methods since they are straightforward and do not require any special calibrations. Further development of PCA might enable the development of spectral libraries useful as a tool to screen for spectral anomalies which could be due to impurities, adulteration or undeclared substances which closely resemble the substance in the library. While this might not 
prove true in all cases, PCA does warrant further investigation for spectroscopic ID tests using other chemically-similar, naturally-derived groups of substances that tend to have variable composition, such as oils, carbohydrates and proteins.

\section{ACKNOWLEDGMENT}

This project was supported in part by the CDER Critical Path and Regulatory Science \& Review Enhancement Programs. This project was supported in part by an appointment (H.K.S) to the Research Participation Program at the Center for Drug Evaluation and Research administered by the Oak Ridge

Institute for Science and Education through an interagency agreement between the U.S. Department of Energy and the U.S. Food and Drug Administration.

\section{DISCLAIMER}

This article reflects the views of the authors and should not be construed to represent FDA's views or policies.

\section{REFERENCES}

[1] K. Meyer, C. Rosa, C. Hischenhuber, R. Meyer, Determination of locust bean gum and guar gum by polymerase chain reaction and restriction fragment length polymorphism analysis, J AOAC Int 84 (2001) 89-99.

[2] S. Tripathy, M.K. Das, Guar gum: present status and applications, J Pharm Sci Innov 2 (2013) 24-28.

[3] Federal Register, Volume 74, Number 64, http://www.gpo.gov/fdsys/pkg/FR-2009-04-06/pdf/E9-7843.pdf, 1/14/2015. [4] A.M. Brustkern, L.F. Buhse, M. Nasr, A. Al-Hakim, D.A. Keire, Characterization of Currently Marketed Heparin Products: Reversed-Phase Ion-Pairing Liquid Chromatography Mass Spectrometry of Heparin Digests, Anal Chem 82 (2010) 9865-9870.

[5] J. Moore, A. Ganguly, J. Smeller, L. Botros, M. Mossoba, Standardisation of non-targeted screening tools to detect adulterations in skim milk powder using NIR spectroscopy and chemometrics, NIR news 23 (2012) 9-11.

[6] X. Li, S. Arzhantsev, J.F. Kauffman, J.A. Spencer, Detection of diethylene glycol adulteration in propylene glycol-Method validation through a multi-instrument collaborative study, J Pharm Biomed Anal 54 (2011) 1001-1006.

[7] A.B. Champagne, K.V. Emmel, Rapid screening test for adulteration in raw materials of dietary supplements, Vib Spectrosc 55 (2011) 216-223.

[8] R.M. El-Abassy, P. Donfack, A. Materny, Visible Raman spectroscopy for the discrimination of olive oils from different vegetable oils and the detection of adulteration, J Raman Spectrosc 40 (2009) 1284-1289.

[9] J.K. Baird, W.W. Smith, A simple colorimetric method for the specific analysis of food-grade galactomannans, Food Hydrocoll 3 (1989) 413-416.

[10] Industrial Minerals, Guar Gum Shortage Impacts Fracking Market, http://am2fenergy.com/wp-content/uploads/Guargum-\%2B-proppants-news-IM-6-12.pdf, 05/26/2015.

[11] Rx 360, Guar Gum Shortage, http://www.rx360.org/AlertsNews/Alerts/GuarGumShortageJanuary2012/tabid/259/Default.aspx, 1/14/2015.

[12] USP-NF, in, USPC Official 5/1/15 NF Monographs: Guar Gum, 2015.

[13] The USP Excipients Stakeholder Forum Meeting \#1 June 7, 2013, Excipient Monograph Modernization, http://www.usp.org/sites/default/files/events/stakeholder_forums/2013/meeting-1/5-excipient-modernization-2013-0607.pdf, 1/14/2015.

[14] J.K. Baird, W.W. Smith, An analytical procedure for gellan gum in food gels, Food Hydrocoll 3 (1989) 407-411. 
[15] USP-NF Online, NF Monographs: Guar Gum, http://www.uspnf.com/uspnf/pub/index?usp=37\&nf=32\&s=2\&officialOn=December 1, 2014, 1/14/2015.

[16] T. Heinze, Erler, U., Klemm, D., Investigations on homogeneous synthesis of carboxy group-containing cellulose derivatives and the determination of the substituent distribution using HPLC, in: Cellulose and Cellulose Derivatives, 1995, pp. 161.

[17] J.D. Rodriguez, B.J. Westenberger, L.F. Buhse, J.F. Kauffman, Quantitative Evaluation of the Sensitivity of LibraryBased Raman Spectral Correlation Methods, Anal Chem 83 (2011) 4061-4067.

[18] J.D. Rodriguez, C.M. Gryniewicz-Ruzicka, S. Arzhantsev, J.F. Kauffman, L.F. Buhse, Rapid Screening Methods for Pharmaceutical Surveillance, in: Science and the Law: Analytical Data in Support of Regulation in Health, Food, and the Environment, American Chemical Society, 2014, pp. 149-168.

[19] K. Carron, R. Cox, Qualitative analysis and the answer box: a perspective on portable Raman spectroscopy, Anal Chem 82 (2010) 3419-3425.

[20] J.D. Rodriguez, B.J. Westenberger, L.F. Buhse, J.F. Kauffman, Standardization of Raman spectra for transfer of spectral libraries across different instruments, Analyst 136 (2011) 4232-4240.

[21] C.M. Gryniewicz-Ruzicka, J.D. Rodriguez, S. Arzhantsev, L.F. Buhse, J.F. Kauffman, Libraries, classifiers, and quantifiers: A comparison of chemometric methods for the analysis of Raman spectra of contaminated pharmaceutical materials, J Pharm Biomed Anal 61 (2012) 191-198.

[22] C.M. Gryniewicz-Ruzicka, S. Arzhantsev, L.N. Pelster, B.J. Westenberger, L.F. Buhse, J.F. Kauffman, Multivariate Calibration and Instrument Standardization for the Rapid Detection of Diethylene Glycol in Glycerin by Raman Spectroscopy, Appl Spectrosc 65 (2011) 334-341.

[23] J.F. Kauffman, J.D. Rodriguez, L.F. Buhse, Spectral Preprocessing for Raman Library Searching, Am Pharm Review 14 (2011) 34-40. 


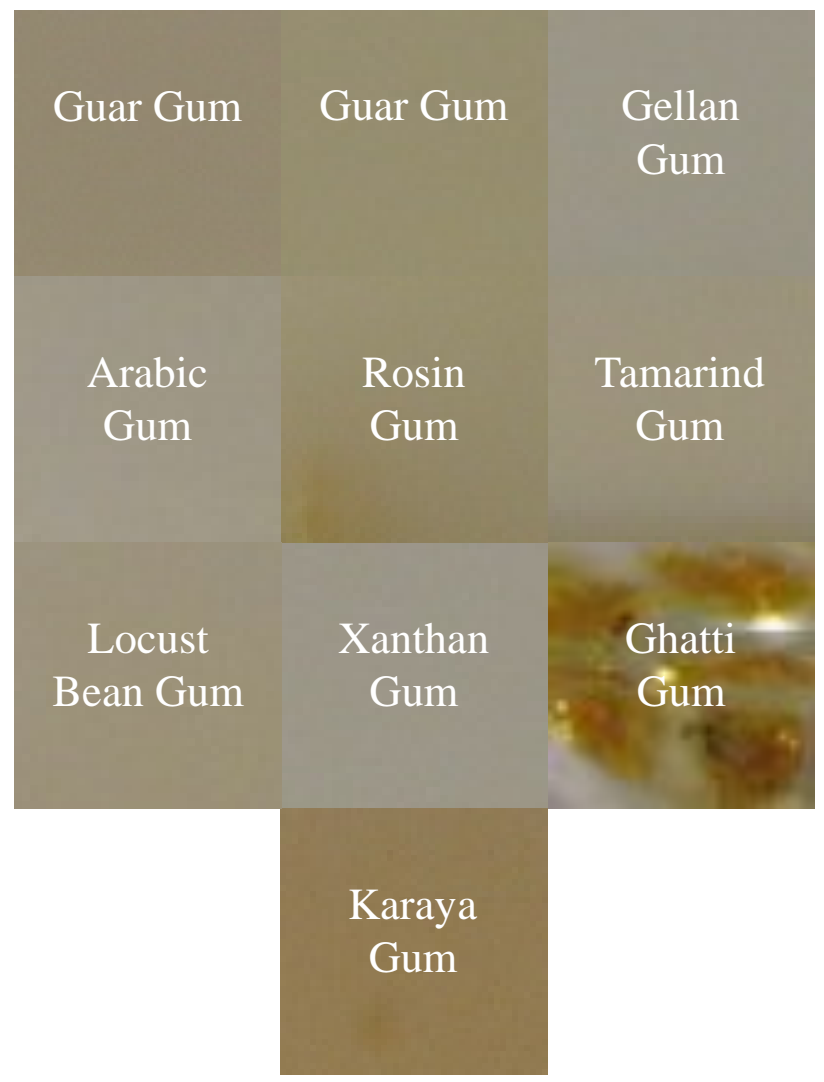

Figure 1. The visual results from the compendial identification test for guar gum (Test A) for the different types of gums used in the study. 

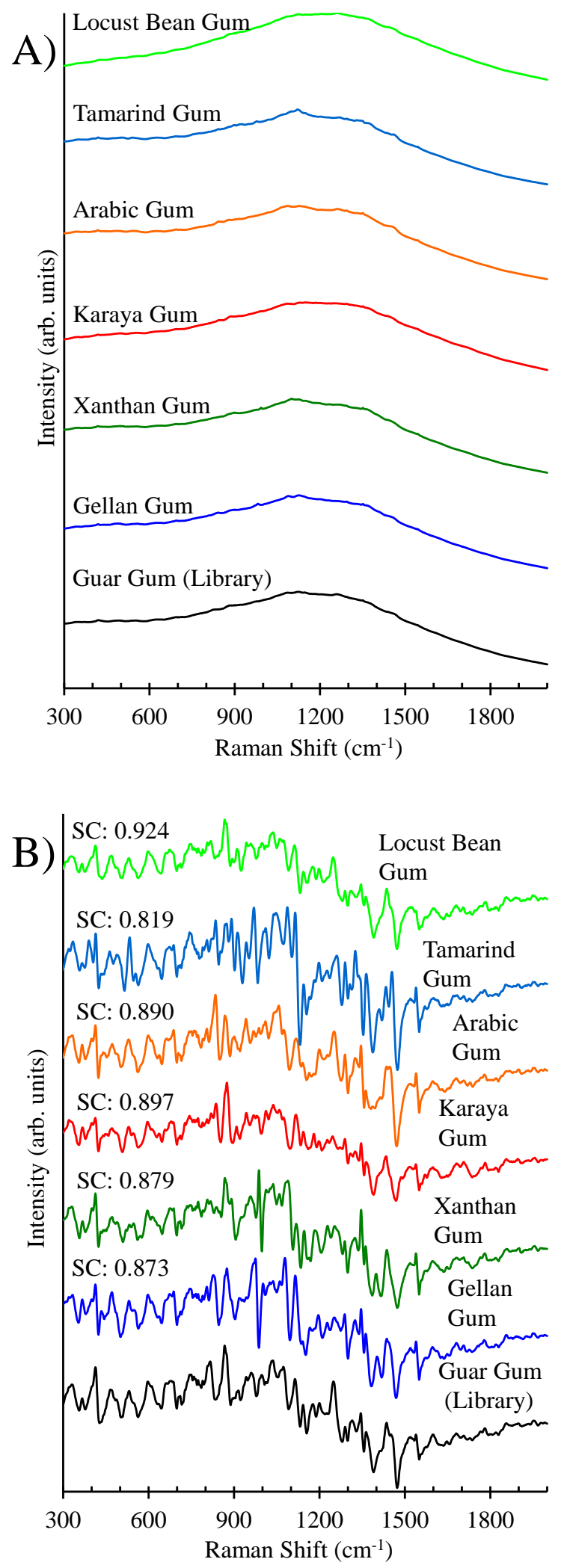

Figure 2. Raman spectra for different types of gums using A) no preprocessing and B) first derivative preprocessing. Each spectrum is the average of the 15 different replicate runs. The spectra have been normalized to their maximum intensity and offset for clarity. 


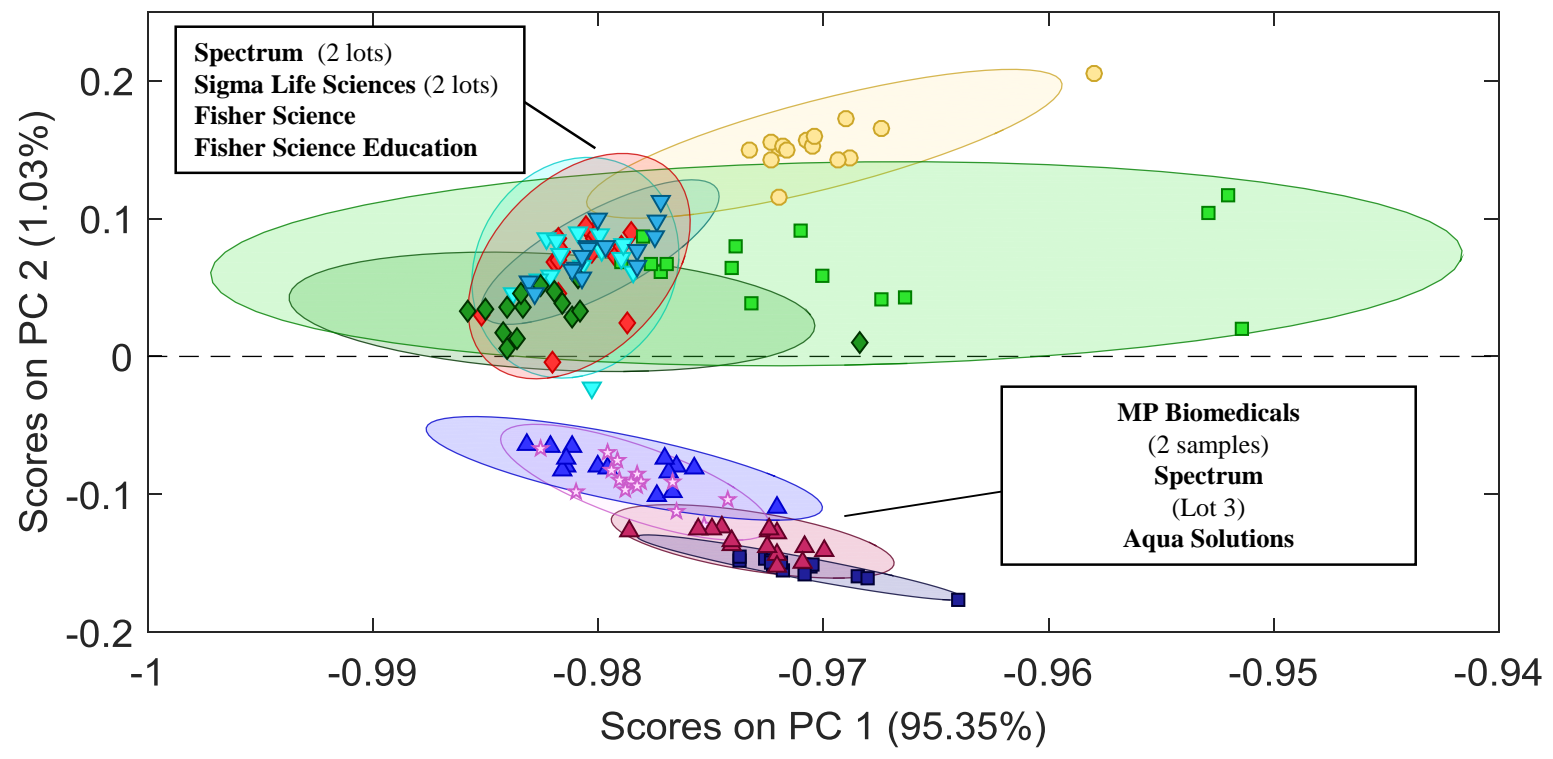

Figure 3. PCA plot of guar gum library samples. A three component model was used for the ten guar gum library samples. All 15 replicate runs were used to generate each class. Two regions of overlap are observed. First derivative preprocessing ( $2^{\text {nd }}$ order, 15 point window) followed by vector length normalization was used. 


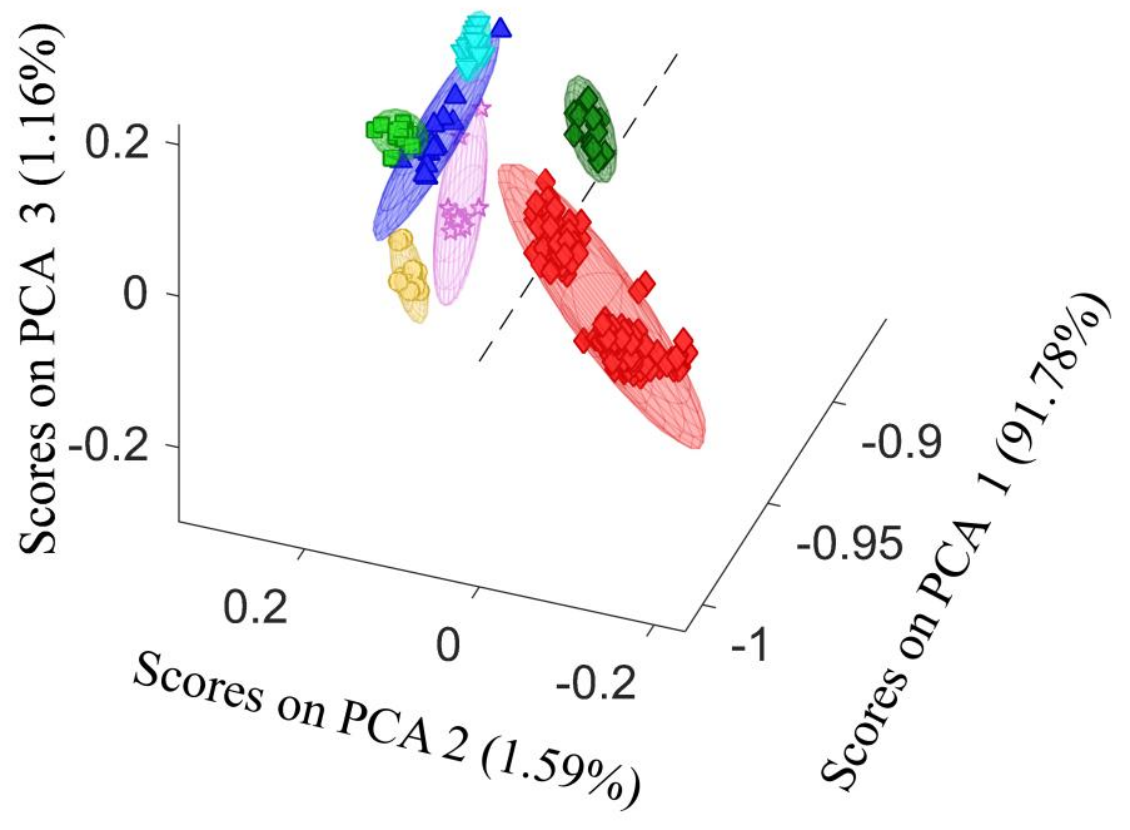

\section{$\diamond$ Library Gellan Xanthan Karaya Arabic Tamarind Locust Bean}

Figure 4. Three component PCA plot of guar gum library samples and other gums. A total of 150 spectra are used for the guar gum class and 15 spectra are used for all other gum classes. All of the nonguar gum samples are clearly discriminated from the guar gum class. First derivative preprocessing $\left(2^{\text {nd }}\right.$ order, 15 point window) followed by vector length normalization was used. 
Table 1. Commercial gum samples used for method development.

\begin{tabular}{|r|c|c|c|c|}
\hline Sample & Grade & Manufacturer & Lot Designation & Type \\
\hline Guar Gum & NF & Spectrum & Lot 1 & Library $^{\mathrm{a}}$ \\
Guar Gum & Research & Sigma Life Sciences & Lot 1 & Library $^{\mathrm{a}}$ \\
Guar Gum & Research & MP Biomedicals & Lot $1^{\mathrm{b}}$ & Library \\
Guar Gum & NF & Spectrum & Lot 2 & Library \\
Guar Gum & Research & MP Biomedicals & Lot $1^{\mathrm{b}}$ & Library \\
Guar Gum & Research & Fisher Science & Lot 1 & Library \\
Guar Gum & Research & Sigma Life Sciences & Lot 2 & Library \\
Guar Gum & NF & Spectrum & Lot 3 & Library \\
Guar Gum & Research & Aqua Solutions & Lot 1 & Library \\
Guar Gum & Research & Fisher Science & Lot 2 & Library \\
Gellan Gum & Research & Alfa Aesar & Lot 1 & Test \\
Xanthan Gum & Research & Sigma Life Sciences & Lot 1 & Test \\
Karaya Gum & Research & Sigma Life Sciences & Lot 1 & Test \\
Arabic Gum & Research & Sigma Life Sciences & Lot 1 & Test \\
Rosin Gum & Research & MP Biomedicals & Lot 1 & Test \\
Tamarind Gum & Research & Tokyo Chemical & Lot 1 & Test \\
Locust Bean Gum & FCC & Spectrum & Lot 1 & Test \\
Ghatti Gum & Research & Pfaltz \& Bauer & Lot 1 & Test \\
\hline
\end{tabular}

${ }^{\mathrm{a}}$ Guar Gum control used for compendial ID test.

${ }^{\mathrm{b}}$ Samples received under different containers. 
Table 2. Results of compendial tests for different types of gums.

\begin{tabular}{|c|c|c|c|c|}
\hline \multirow[b]{3}{*}{ Sample } & \multirow{3}{*}{$\begin{array}{c}\text { Compendial } \\
\text { ID } \\
\text { Decision } \\
\end{array}$} & \multicolumn{3}{|c|}{ Spectral Based ID } \\
\hline & & \multicolumn{2}{|c|}{ Raman } & \multirow{2}{*}{$\begin{array}{l}\text { Raman } \\
\text { PCA ID } \\
\end{array}$} \\
\hline & & SC & Decision $^{a}$ & \\
\hline Arabic & Fail & 0.890 & Fail & Fail \\
\hline Gellan & Pass & 0.873 & Fail & Fail \\
\hline Karaya & Fail & 0.897 & Fail & Fail \\
\hline Tamarind & Fail & 0.819 & Fail & Fail \\
\hline Xanthan & Fail & 0.879 & Fail & Fail \\
\hline Locust Bean & Fail & 0.924 & Fail & Fail \\
\hline Rosin & Fail & $\mathrm{n} / \mathrm{a}$ & $\mathrm{n} / \mathrm{a}$ & $\mathrm{n} / \mathrm{a}$ \\
\hline Ghatti & Fail & $\mathrm{n} / \mathrm{a}$ & $\mathrm{n} / \mathrm{a}$ & $\mathrm{n} / \mathrm{a}$ \\
\hline
\end{tabular}

${ }^{a}$ A 0.95 threshold used for pass. 